Research Article

\title{
The Characteristic Transient Response of a Pressurized Cantilever Pipe Subjected to Transverse Impact at Its Tip
}

\author{
Feng Liu (D), Yuchao Yang, and Yuelei Wu \\ Shandong University of Science and Technology, Shandong Provincial Key Laboratory of Civil Engineering Disaster \\ Prevention and Mitigation, Qingdao 266510, China
}

Correspondence should be addressed to Feng Liu; Feng.Liu@sdust.edu.cn

Received 19 August 2018; Revised 4 November 2018; Accepted 2 May 2019; Published 22 May 2019

Academic Editor: Miguel Neves

Copyright $(92019$ Feng Liu et al. This is an open access article distributed under the Creative Commons Attribution License, which permits unrestricted use, distribution, and reproduction in any medium, provided the original work is properly cited.

This paper describes an experimental study on the pure bending mechanical behavior of a pressurized pipe and adoption of a measured moment-curvature relationship under different working conditions in numerical simulations for transient pipe-whip prediction. To describe the effects of pipe contents and internal pressure, the governing equations were derived based on large deformation theory. Bending moment and axial force were uncoupled in the constitutive equation, and an experiment-based relationship between moment and curvature was adopted. The numerical simulations show that the present model can simulate the mechanical processes of elasticity, plastic hardening, and softening behavior in the initial, middle, and late stages of whole response, respectively. In addition, it was shown that kinks may occur at several positions along an empty cantilever pipe due to the collapse of sections under intense dynamic loading. However, this behavior did not occur for the full pressurized pipe, indicating that the contents and internal pressure are able to effectively impede the partial flattening of the pipe section, improving its critical curvature and changing its plastic dynamic response behavior.

\section{Introduction}

Pipes for conveying high-energy and high-pressure fluids are widely used in nuclear power plants and petrochemical facilities. Under collision and other intense loading conditions, such components may undergo large flexural deformation, possibly resulting in structural collapse and catastrophic chain reactions. Typical engineering cases include pipe-whip caused by sudden rupture of a high-energy piping system after accidental impact $[1,2]$.

Two modes of deformation are possible when a thin circular tube is subjected to bending loads. In the first mode, bending induces ovalization of the tube cross section. The growth of ovalization reduces the bending rigidity of the tube, causing the flexural capacity to decrease when this reduction surpasses the strain hardening of the material. This phenomenon, first discovered by Brazier, is often referred to as the Brazier effect [3]. In contrast to the well-known elasticplastic hardening phenomenon, this process is always called softening behavior [4]. The second deformation mode of a tube under bending involves buckling on the compressed side of the tube due to excessive compressive stress. The experimental studies of Yu et al. [3], Reid et al. [4, 5], and Prinja and Chitkara [6] have recorded the softening behavior of a cantilever pipe beam during the deformation process due to individual sections exceeding the critical curvature. Based on the moment-curvature relationship obtained by four-point bending experiments for the empty pipe and the large deflection governing equation based on a generalized constitutive equation, Reid et al. [5] used a 2D model of in-plane deformation and a 3D model including out-of-plane deformation to numerically simulate the elastic-plastic dynamic response of a cantilevered empty pipe. Under intense load, kinks appeared due to softening of local sections. At the same time, based on several experimental results and simple theoretical derivations, Reid et al. $[4,5]$ and Prinja and Chitkara [6] proposed that the critical curvature for plastic hardeningsoftening behavior can be estimated by 


$$
\kappa_{\mathrm{cr}}=C\left(\frac{H}{R^{2}}\right),
$$

where $H$ is the wall thickness of the pipe and $R$ is the radius of the middle surface of the tubular section of the undeformed pipe. The value of $C$ depends on both the material properties and the $H / R$ ratio; it needs to be selected in combination with experiments. Meanwhile, Reddy [7] conducted some experiments on steel and aluminum tubes subjected to pure bending and found that the critical strains at buckling under both bending and axial compression bear a similar relationship to the $H / R$ ratio. By adopting bilinear and Ramberg-Osgood relationships between stress and strain, respectively, Reddy [8] and Gellin [9] extended Brazier's analysis on ovalization into the plastic range and predicted the maximum bending moment and the point of bifurcation for plastic ripples and ovalization. For thick pipes, such as those involved in pipe-whip problems, Munz and Mattheck [10] proposed an analytical solution to calculate the critical bending moment for plastic collapse initiation by considering the vertical component of the axial bending stress as the primary cause of section flattening. Prinja and Chitkara performed further analysis and a series of four-point bending tests on seamless carbon steel pipes $[6,11]$. Meanwhile, in connection with numerical calculations of the hardening-softening behavior of pipes in plastic bending, Prinja and Chitkara [11] investigated the behavior following cross-sectional flattening of relatively thick-walled pipes using the finite element code ABAQUS using four different elements: elbow, shell, solid, and generalized plane strain (GPS) elements. Zhang and Yu [12] presented a relatively simple analysis of ovalization of tubes under pure elastic-plastic bending based on some deformation assumptions; their numerical example showed good agreement with those obtained from more complicated models. Kyriakides and Ju [13] performed a study of this problem involving long aluminum 6061-T6 tubes with 11 different diameter-to-thickness $(D / t)$ ratios ranging from 60.5 to 19.5 . They observed that thinner tubes were inclined to develop short-wavelength periodic ripples on the compressed side of the tube and to collapse soon after local buckling, but the thicker tubes always exhibited a limit-load instability as a direct consequence of ovalization. The same authors [14] also developed a numerical procedure to simulate the interaction between these two sorts of instability. Limam et al. $[15,16]$ used small-scale seamless stainless steel tubes with $D / t$ of about 52 in a systematic investigation of the response and collapse of long tubes bent under internal pressure. Pressure levels between 0 and 0.75 times of the yield pressure $P_{0}=\left(2 \sigma_{0} t / D_{0}\right)$ were considered. They showed that internal pressure can significantly stabilize the tube by inhibiting ovalization. The results of Houliara and Karamanos [17] also support the conclusion that internal pressure can significantly reduce pipeline ovalization. In addition, Mathon and Limam [18] found that internal pressure can reduce the effect of geometric imperfections.

Now turning to the field of impact behavior of the pressurized pipe, many articles have been written about the impact behavior of pipes, whether empty, filled, or pressurized, and some theoretical predictions and empirical equations are available for design purposes $[19,20]$. Palmer et al. [21] used a seamless circular steel tube with a length of $1.8 \mathrm{~m}$, outer diameter of $150 \mathrm{~mm}$, and wall thicknesses $\mathrm{H}$ of $7.2 \mathrm{~mm}, 11 \mathrm{~mm}$, and $18 \mathrm{~mm}$. Using three kinds of bullets-flat head, cone head, hemispherical head-to simulate the impact object, they conducted a systematic experiment on pressurized piping. The experimental results show that pipe contents reduce the critical impact failure speed of the pipe by about $25 \%$ and that the failure speed is not sensitive to changes in internal pressure. Johnson et al. [22] used a conical bullet to perform a penetration experiment on a circular pipe placed on a flat surface, finding a perforation speed of $165 \mathrm{~m} / \mathrm{s}$. This perforation speed is greater than the critical perforation speed required by a standalone pipe, indicating that support conditions affect the perforation speed of a pipe. To study the influence of supports, missile mass, and missile radius on pipe penetration energy and failure mode, Corbett et al. [23] conducted experiments on pipes with diameters of $51 \mathrm{~mm}$, $102 \mathrm{~mm}$, and $153 \mathrm{~mm}$ using hemispherical bullets with masses of $13.6 \mathrm{~g}, 67.9 \mathrm{~g}$, and $105 \mathrm{~g}$. The study also found that different missile shapes will induce different modes of failure. Jones and Birch [24] carried out pipe impact tests with a wedgeshaped projectile, and the pipe did not perforate, instead of cracking at the impact position or damaging the support. However, Schwer et al. [25] used hemispherical bullets to test the pipe wall and perforation did occur. It is worth noting that to simplify the problem, some experimental and theoretical research works on the dynamic response of cantilever pipes ignore the contents and pressure in the pipe. In fact, in an experimental study on the impact mechanical behavior of fixed-end pipe, Jones et al. [2, 24, 26] used a drop hammer system for a series of impact tests on a circular pipe with diameter of $60 \mathrm{~mm}$, wall thickness of $1.7 \mathrm{~mm}$, and span of $600 \mathrm{~mm}$ filled with nitrogen at different pressures. The wedge impactor used had a mass of $21.5 \mathrm{~kg}$, maximum impact velocity of $13.6 \mathrm{~m} / \mathrm{s}$, and under nitrogen pressures of $0 \mathrm{MPa}$, 6.63 MPa, 9.93 MPa, and 13.27 MPa. Accounting for differences in failure positions and critical impact energy under lateral impact, Jones divided failure into two modes: local failure at the impact point and global failure caused by bending deformation. The experimental results also show that the local and global deformation ratios of the pipe will decrease and increase, respectively, with an increase in pipe pressure. Also, as internal pressure increases, the critical impact energy of the corresponding pipe gradually decreases. Palmer et al. [21, 27], Nishida and Tanaka [28], and Chen and Shen [29] found that a full pipe experienced smaller local deformations than an empty pipe, regardless of pressure. At the same time, Jones and Birch [2], Shah [30], and Lu et al. $[31,32]$ conducted experiments on pipes filled with nitrogen and water at different pressures and found that an increase in pressure caused a shift from the local failure mode at the impact location to the global failure mode while causing a reduction in failure energy. Although the conclusions based on different experimental equipment and the mechanisms of dynamic pipe failure still need to be more deeply researched, pipe contents and pressure profoundly affect the pipe dynamic behavior [2]. 
When a cantilever pipe is subjected to dynamic plastic bending, local buckling or cross-sectional shape distortion usually occurs at the fixed boundary [3-5]. The inertial effect will significantly affect the response process. How does the contents of the pipe, or the internal pressure, affect its dynamic behavior? Despite the wide applicability to engineering questions, little analysis of this particular problem has been performed so far, which will be the main objective of the present study.

The bending moment-curvature relationships are measured by a designed device in Section 2. The numerical methods are described in Section 3 based on large deformation theory and measured general constitutive models. Numerical results are shown in Section 4 to demonstrate the success of the proposed method, which is followed by conclusions.

\section{Pure Bending Experiments for Pipes}

2.1. Experimental Equipment and Working Conditions. All of the pipe specimens were cut from seamless circular steel tubes of outer diameter $D=25 \mathrm{~mm}$ and wall thickness $H=1 \mathrm{~mm}$ in their as-received condition. To ensure accuracy, the material tensile test is carried out by directly taking a standard test piece from the base metal. The stress-strain relationship of the pipe is shown in Figure 1. Calculated by standard experimental methods, the material has elasticity modulus of $208 \mathrm{GPa}$, yield strength of $480 \mathrm{MPa}$, and tensile strength of $551 \mathrm{MPa}$.

Traditional experiments to test bending performances of pipes are usually carried out with three-point or four-point bending tests for solid-section components [33]. However, for thin-walled pipes, such experimental methods usually cause large local plastic deformation at the loading site, lead to partial collapse of the structure before the global bending failure occurs, and thus fail to predict the global bending performance of the pipe.

To overcome these shortcomings, a circular thin-walled pipe pure bending device as shown in Figure 2 was designed. This device consists of two free-turning sprockets with a radius of $0.15 \mathrm{~m}$ mounted on two stiff support beams. A heavy chain runs around each sprocket and is connected by steel strand to a tension sensor and double-acting jack, forming a closed loop. Each test specimen is connected at both ends to a hollow steel rod with an outer diameter that just fits into the inner diameter of the pipe. The end of the steel rod connected to the specimen can be inserted $15 \mathrm{~cm}$ into the pipe. The assembly was mounted onto the bending device and the steel rod passes smoothly between four U-groove rollers. In addition, two displacement sensors with a range of $250 \mathrm{~mm}$ are connected to the chains on both sides to record the displacement, which equals to the length of the arc through which the sprocket turns. The machine was activated by contracting the lower double-acting jack with the hydraulic servo loading device and simultaneously extending the other, causing rotation of the sprockets and the hard rods. Compared to the traditional four-point bending instrument, the specimen was purely bent and

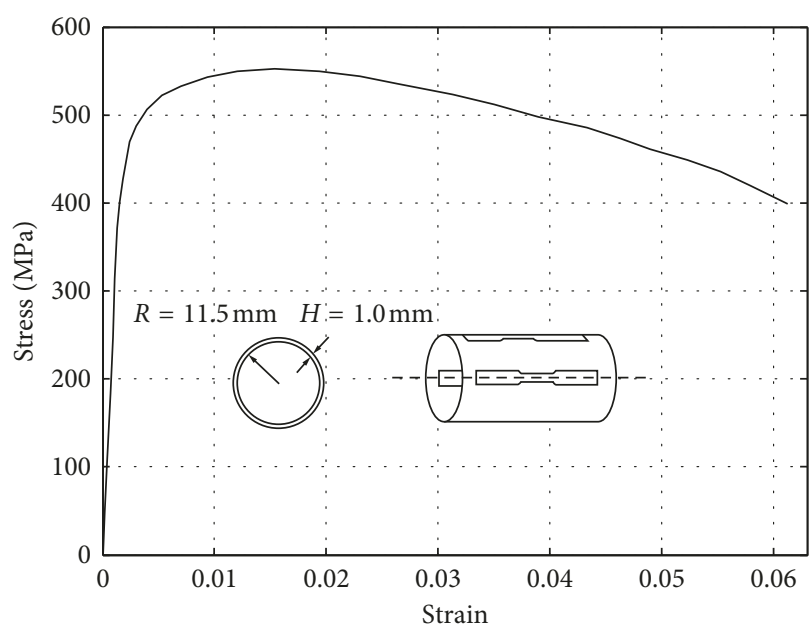

Figure 1: Recorded stress-strain curve of the pipe material.

deformed during the whole test process due to the rotation of the hard rods at both ends.

Corresponding to the illustration of the pure bending device for pipe in Figure 2, the measurement of the force and displacement is demonstrated in Figure 3. Figures 3(a) and $3(\mathrm{~b})$ are the initial and working conditions of the bending process, respectively. In Figure 3, $a$ is the half-length of the pipe pure bend section; $R_{\mathrm{S}}$ is the radius of the sprocket; $F_{1}$ and $F_{2}$ are the tensions of the two sprockets; $L_{1}$ and $L_{2}$ are the arc lengths of the two sprocket circles; $\theta_{1}$ and $\theta_{2}$ are the center angles of the two sprocket circles rotating through the arc lengths $L_{1}$ and $L_{2}$; and $R^{\prime}$ is the radius of curvature of the corresponding pipe when the sprocket rotates the arc length $L$.

Given the configuration shown in Figure 3(b), the bending moment and curvature can be calculated by

$$
\begin{gathered}
M=\left(F_{1}+F_{2}\right) \cdot \frac{R_{\mathrm{S}}}{2}, \\
\kappa=\frac{L_{1}+L_{2}}{2 a \cdot R_{\mathrm{S}}},
\end{gathered}
$$

where $F_{1}$ and $F_{2}$ and $L_{1}$ and $L_{2}$ are force and displacement measured by sensors during the test process.

To fill and pressurize the pipe, a precision snap ring and high-strength carbon steel ferrule are used to form the joint between the pipe and the steel bar. The compression of the snap ring produces a radial force, ensuring a good fit and sealing the pipe contents. The pressure is recorded and monitored by a pressure sensor mounted on the side of the nonpressing end steel rod. The hydraulic control device and the load, displacement, and pressure sensors are connected to the Donghua DH-5929 dynamic acquisition system. The data acquisition frequency is set as $5 \mathrm{~Hz}$ in present test.

To investigate the influence of different pipe contents on bending behavior, water and air are selected in present test since they have similar properties to oil and natural gas used in the actual pipe engineering. In addition, to increase contrast and investigate the influence of a dense medium on the bending behavior of the pipe, sand is also adopted; it can be considered as the transition state between empty pipe and 

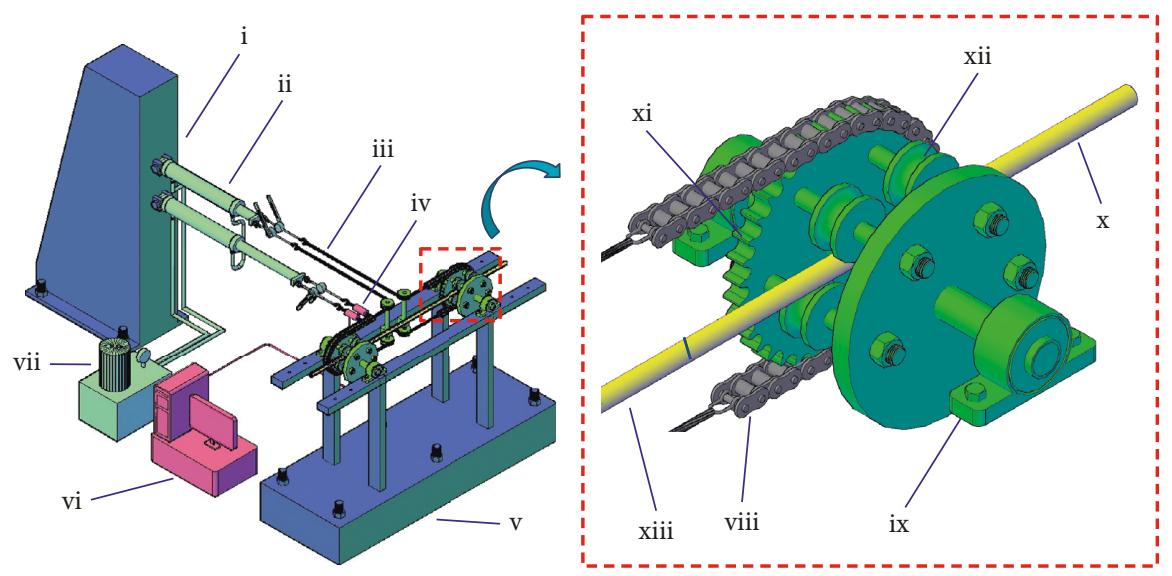
(i) Reaction support
(ii) Double acting jack
(iii) Steel strand
(iv) Tension sensor
(v) Support

(vi) Acquisition system

(xi) Sprocket

(vii) Hydraulic driver

(xii) U-shaped roller

(viii) Chain

(xiii) Tube

(ix) Base bearing

(x) $\operatorname{Rod}$

Figure 2: Demonstration of the pure bending device for pipes.

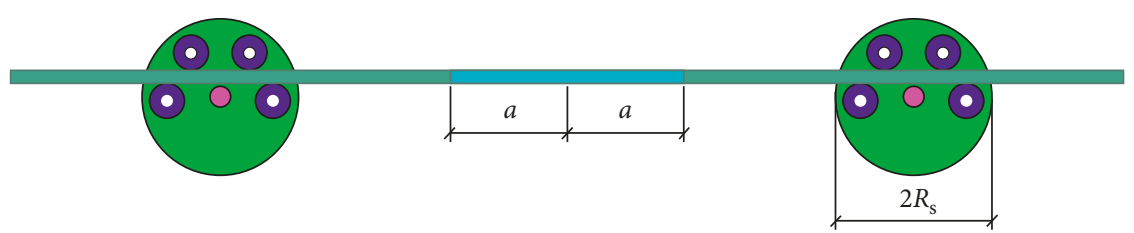

(a)

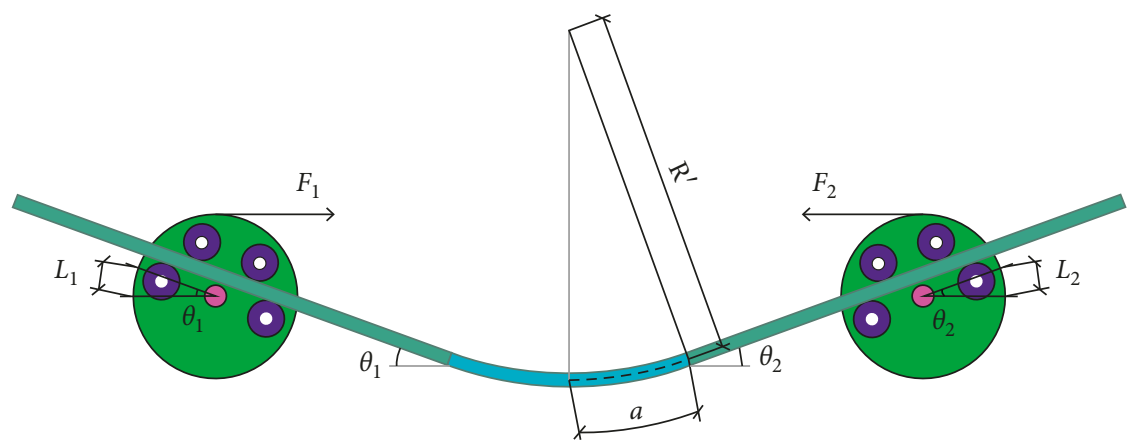

(b)

FIGURE 3: Illustration for measurement of force and displacement: (a) initial and (b) during the test.

a circular solid section. According to Jones and Birch [2], the critical pressure of the pipe can be predicated by $p_{y}=\sigma_{y} H / R$, so three pressure levels, i.e., $\phi_{p}=p / p_{y}=$ $12.5 \%, 25 \%, 37.5 \%$, are selected for present experiment. Meanwhile, the test on empty pipes is also conducted for comparison. In addition, to examine the influence of pipe contents aside from internal pressure, experiments on pipes filled with water at zero internal pressure is performed simultaneously. Sand does not exert internal pressure due to its material properties. The relevant experimental parameters are listed in Table 1.

2.2. Experimental Observations. The original and deformed configurations of a pipe tested by the device described in
TABLE 1: Test parameters of pressurized pipes for pure bending.

\begin{tabular}{lccc}
\hline $\begin{array}{l}\text { Pipe length } \\
L_{0}(\mathrm{~mm})\end{array}$ & $\begin{array}{c}\text { Pure bend } \\
\text { length } 2 a(\mathrm{~mm})\end{array}$ & $\begin{array}{c}\text { Medium } \\
\text { in pipe }\end{array}$ & $\begin{array}{c}\text { Internal } \\
\text { pressure }(\mathrm{MPa})\end{array}$ \\
\hline 750 & 450 & Water, air, sand & $0,5,10,15$ \\
\hline
\end{tabular}

Figure 4 are shown in Figure 5. At the same time, the kink located in the interior of the deformed specimen is highlighted. According to the comprehensive experimentally observed phenomenon, the bending deformation occurs uniformly in the pure bend section of the pipe at the initial stage of load application, and the pipe deformation mainly manifests as section transitions from circular to flat. When the load increases, the global bending deformation almost 


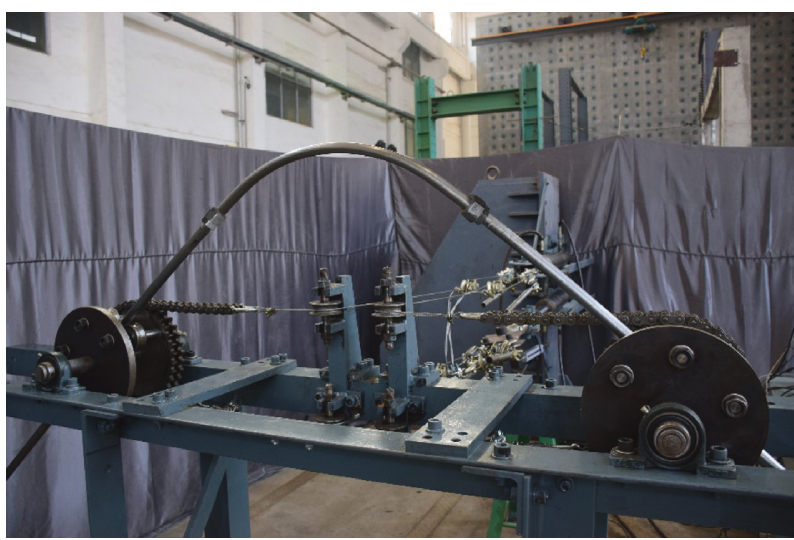

Figure 4: Photos of the pure bending device.

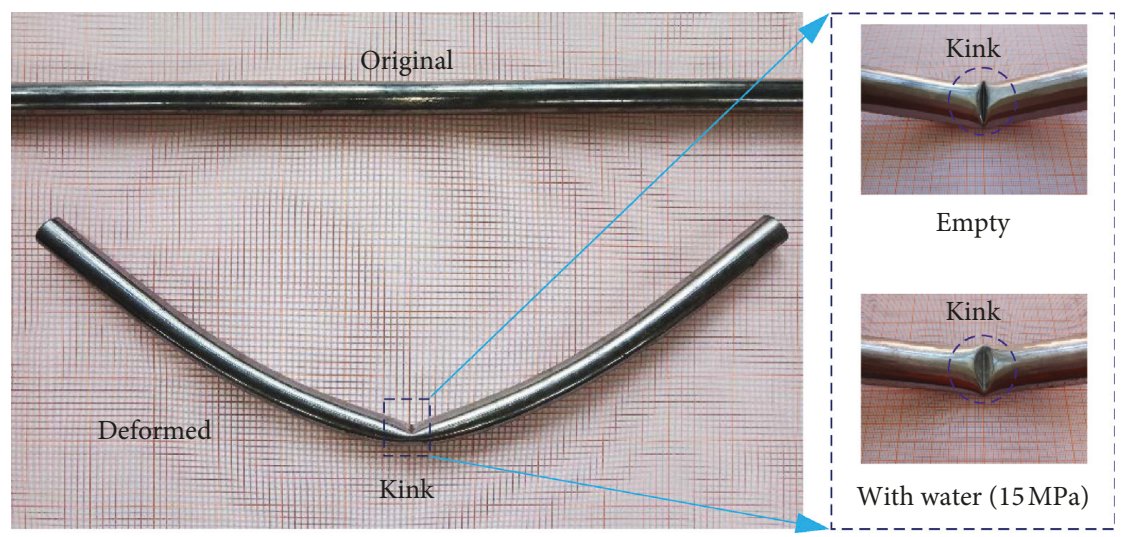

Figure 5: Deformed configuration of the specimen.

stops and plasticity rapidly develops on a shorter section in the interior of the pure curved section, forming a kink, which was observed in pipe whipping experiments observed by $\mathrm{Yu}$ et al. [3] and Reid et al. [4, 5]. Then, the bending moment value rapidly decreases followed by the pipe losing its bending capacity. This phenomenon is unique for the thinwalled pipes and was not found in the four-point bending experiment of the traditional solid-section component.

Unlike in the solid-section component, the gradual flattening or ovalization of the pipe section is a distinctive deformation mechanism and should be a crucial consideration for the theoretical prediction of its flexural behavior. Figure 6 shows the contrast in the original and deformed cross section of the pipe when filled with sand, water (pressure $15 \mathrm{MPa}$ ), and nothing. It was found that the three specimens all show an obvious flattening tendency under bending, which indicates that even if the pipe is filled or pressurized, the cross-sectional shape changing under bending is unavoidable. However, the empty pipe is flattened the most, the sand-filled pipe the least, and the water-filled specimen somewhere in between. Therefore, a filled pipe can effectively resist a change in cross-sectional shape, affecting its flexural behavior.

During the loading process, the lower jack is uniformly contracted to rotate the sprocket slowly at a constant speed and then apply pure bending to both ends of the pipe. A typical relationship between tension and displacement is illustrated in Figure 7. It shows that due to the elastic properties of the material, the load increases rapidly with the displacement increase at the beginning and reaches a peak after short-term hardening followed by a rapid drop as deformation increases. The entire response curve has a remarkable characteristic as elastic in early stage, plastic hardening in medium stage, and postsoftening in last stage. This is consistent with the observation by Yu et al. [3], Prinja and Chitkara [6], and Reid et al. [4] for thin-walled empty pipes.

According to Boyle's rule, when the temperature change is negligible, the variation of pressure can be used to predict the change of the specimen's volume. To this end, Figure 8 records the time history of internal pressure for a pipe with gas at an initial pressure of $15 \mathrm{MPa}$. It was shown that the pressure increases along the increasing load and terminates at $15.35 \mathrm{MPa}$, indicating that the volume of the pipe is gradually reduced due to bending deformation. Simple calculations show that the global bending of pipe and the local flattening of individual sections cause its volume to drop by approximately $2.33 \%$ which implies that even if the pipe is filled, volume change still occurs, but the response mechanism is still dominated by the transformation of global configuration. This observation provides a foundation for present theoretical hypothesis and formula derivation. 


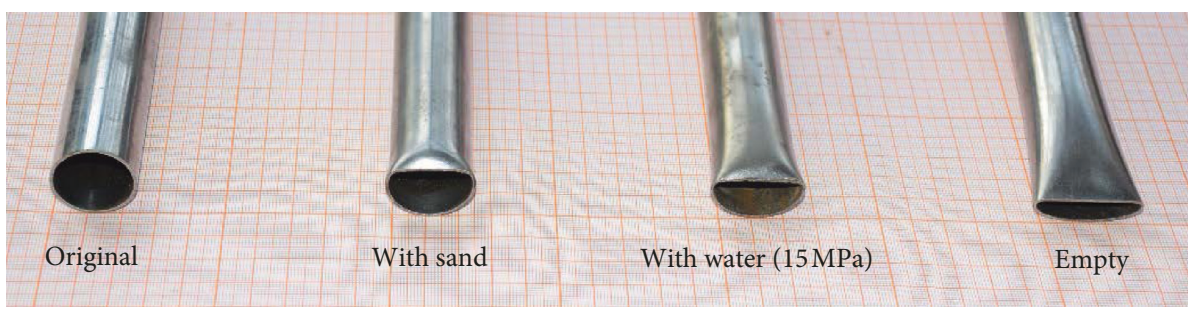

Figure 6: Comparison of the deformed section with different media.

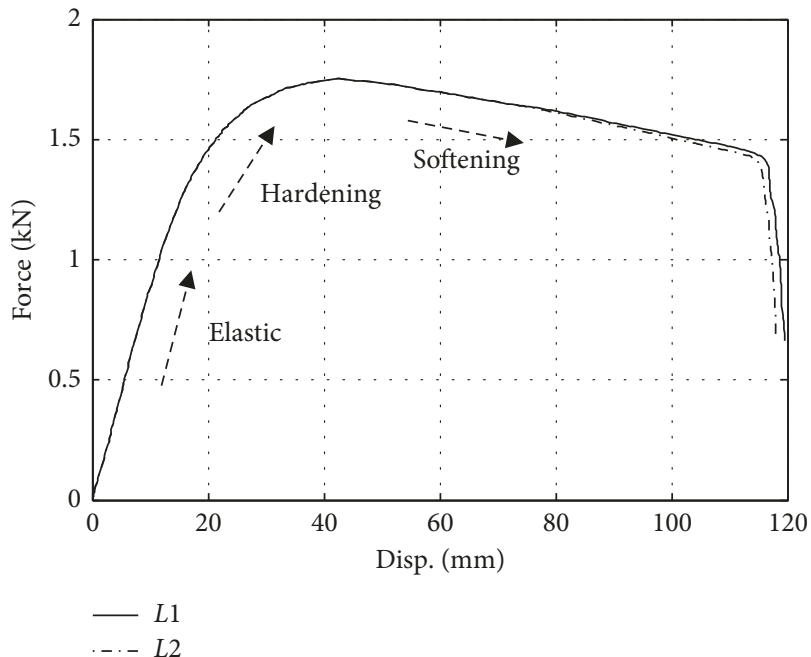

FIGURE 7: Evolution of tension against displacement of sprocket wheel for the empty pipe.

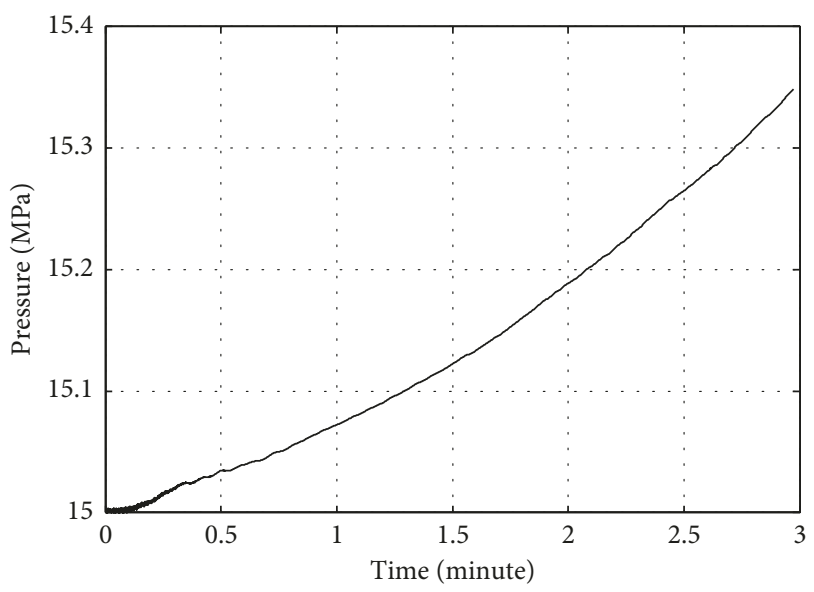

FIGURE 8: Time history of internal pressure during the bending.

2.3. Moment-Curvature Relationship. Generally, the moment-curvature $(M-\kappa)$ relationship is always used to evaluate the flexural performance of the components. To examine the influence of pipe contents, Figure 9 compares the $M-\kappa$ curve for pipes filled with water, air, and sand with zero internal pressure. It can be seen that in the case of the water-filled pipe, the peak bending moment and critical curvature slightly increased from $262 \mathrm{~N} \cdot \mathrm{m}$ and $1.25 \mathrm{~m}^{-1}$ for the empty pipe to $278 \mathrm{~N} \cdot \mathrm{m}$ and $1.52 \mathrm{~m}^{-1}$. However, even with improved bending resistance, the pipe shows obvious

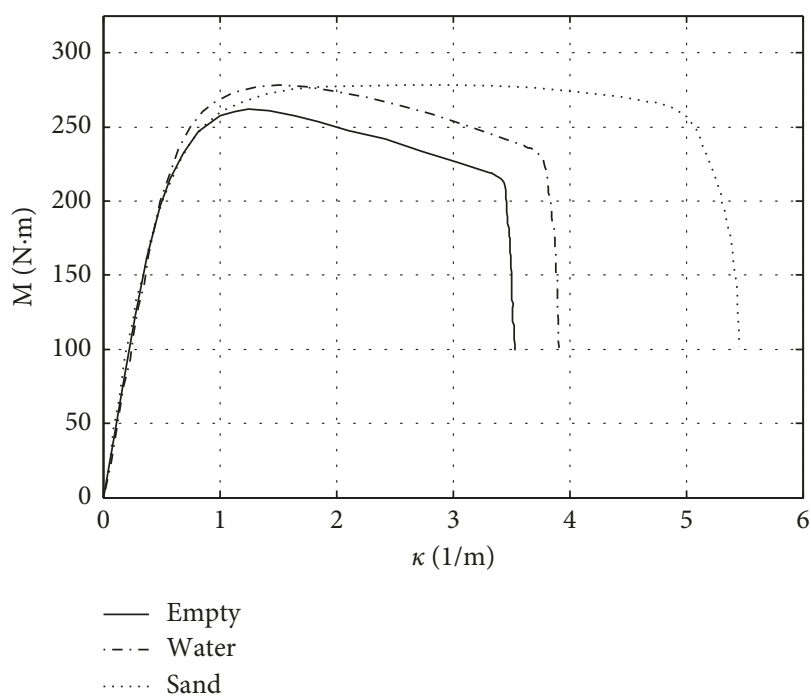

FIgURE 9: Recorded $M-\kappa$ curves of pipe with different media and no pressure.

softening behavior after later plastic deformation. Comparatively, a pipe filled with sand exhibits very different bending behavior; it does not have a much larger peak bending moment than a water-filled one. However, its bending moment does not decrease immediately after reaching the critical curvature but remains relatively constant between 1.9 and $4.0 \mathrm{~m}^{-1}$, which means that the pipe can maintain bending resistance during a large rotation similar to solid components. So, internal sand can effectively prevent the formation and development of local deformations of a pipe section under bending.

In addition, to investigate the influence of internal pressure and exclude the effect of the contents, Figure 10 shows the $M-\kappa$ curve at three pressures of water and air, respectively. It was observed that in a water-filled pipe, the pressure inside the $15 \mathrm{MPa}$ pipe will increase peak bending moment and critical curvature to $400 \mathrm{~N} \cdot \mathrm{m}$ and $3.2 \mathrm{~m}^{-1}$, respectively, from the no-pressure $278 \mathrm{~N} \cdot \mathrm{m}$ and $1.52 \mathrm{~m}^{-1}$. For the air-filled case, an internal pressure of $15 \mathrm{MPa}$ can increase the peak bending moment and critical curvature from $262 \mathrm{~N} \cdot \mathrm{m}$ and $1.25 \mathrm{~m}^{-1}$ in an empty pipe to $343 \mathrm{~N} \cdot \mathrm{m}$ and $2.7 \mathrm{~m}^{-1}$. These indicate that the peak bending moment and critical curvature of the pipe increase as the internal pressure increases. However, regardless of pressure, the bending resistance of the pipe rapidly decreases and exhibits a softening behavior after reaching the critical curvature. There was no ability to resist bending similar to the sand- 


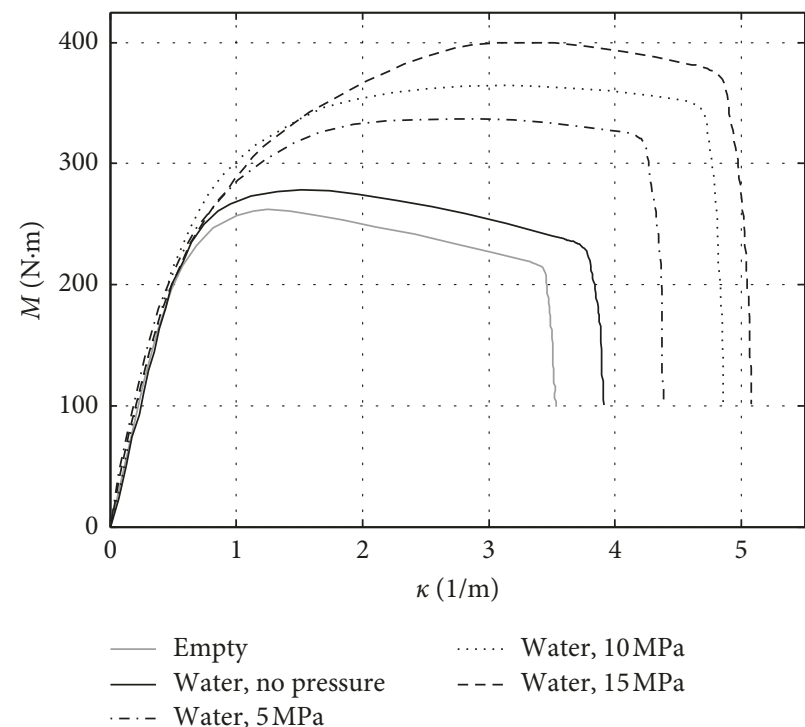

(a)

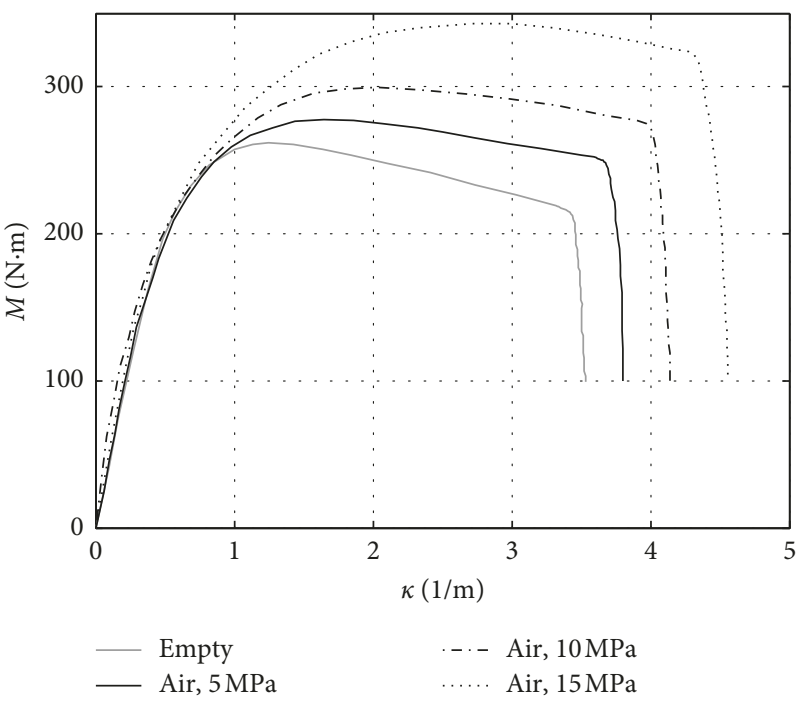

(b)

Figure 10: $M-\kappa$ curves of the pipe with different internal pressures (a) with water and (b) with air.

filled pipe. Even so, the increase in critical curvature may significantly reduce kink behavior occurring during the process of pipe whipping.

To visually compare the bending resistance of different contents at the same internal pressure, Figure 11 summarizes the $M-\kappa$ curve at three pressures in both water- and air-filled pipes. When the internal pressure is $5 \mathrm{MPa}$, the critical curvature and peak bending moment of the air-filled pipe are $1.65 \mathrm{~m}^{-1}$ and $277 \mathrm{~N} \cdot \mathrm{m}$ compared to $2.9 \mathrm{~m}^{-1}$ and $336 \mathrm{~N} \cdot \mathrm{m}$ for the water-filled pipe. This trend extends as expected when the pressure reaches $15 \mathrm{MPa}$; in this case, the critical curvature and peak bending moment of the air-filled pipe are $2.7 \mathrm{~m}^{-1}$ and $343 \mathrm{~N} \cdot \mathrm{m}$ compared to $3.2 \mathrm{~m}^{-1}$ and $400 \mathrm{~N} \cdot \mathrm{m}$ for the waterfilled pipe. The comparisons indicate that under the same internal pressure, the critical curvature and peak bending moment are much better for the water-filled pipe than for the air-filled pipe and implies that the content of the pipe is another important factor affecting its bending behavior.

\section{Numerical Method for Transient Response Prediction of a Pressurized Pipe}

3.1. Governing Equations. The mechanical model of a pressurized cantilever pipe subjected to the concentrated impact force $F(t)$ at its tip can be simplified as shown in Figure 12. The pipe radius, wall thickness, and length are $R$, $H$, and $L$, respectively.

Consider the microsection of the pressurized pipe shown in Figure 13, which is deformed from the initial $i^{*} j^{*}$ to $i j$. The correspondence between the internal force and the deformation can be expressed by

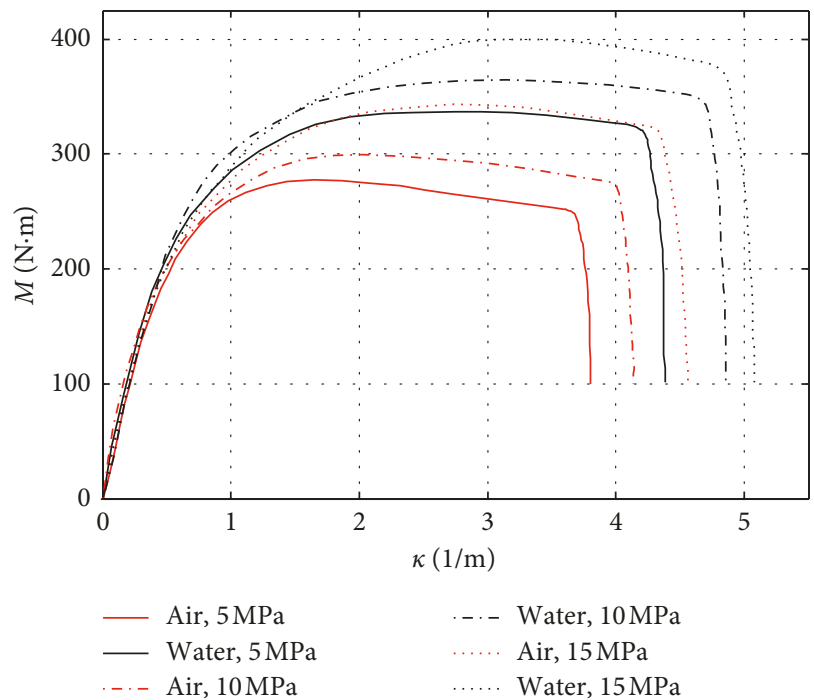

FIGURE 11: $M$ - $\kappa$ curves of the pipe with water and air and different levels.

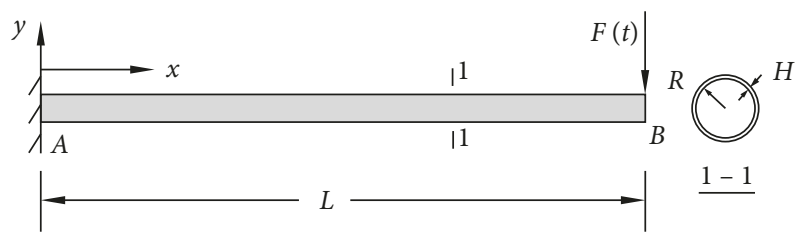

FIGURE 12: Model of the pressurized cantilever pipe subjected to impact at its tip. 


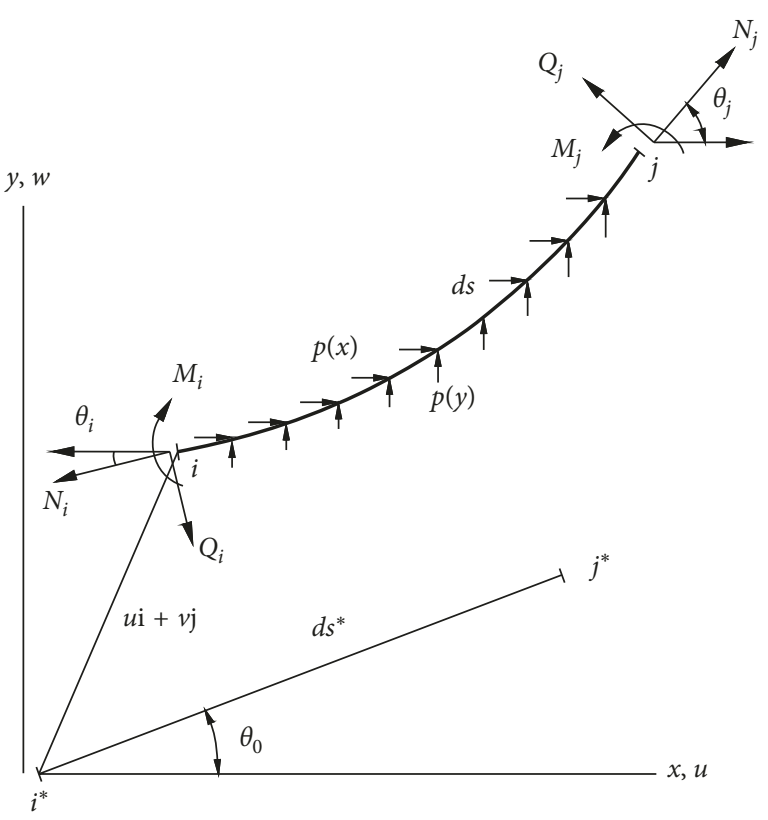

FIgURE 13: Typical segment of structure under configuration of large deformation.

$$
\left.\begin{array}{c}
N_{j}=N_{i}+\frac{\partial N}{\partial s} d s, \\
Q_{j}=Q_{i}+\frac{\partial Q}{\partial s} d s, \\
M_{j}=M_{i}+\frac{\partial M}{\partial s} d s, \\
u_{j}=u_{i}+\frac{\partial u}{\partial s} d s \\
v_{j}=v_{i}+\frac{\partial v}{\partial s} d s, \\
\theta_{j}=\theta_{i}+\frac{\partial \theta}{\partial s} d s .
\end{array}\right\} .
$$

From the dynamic balance of the microsegment, the kinematic differential equation of the pipe segment can be derived as [34]

$$
\left.\begin{array}{l}
\frac{\partial}{\partial s}(N \cos \theta)-\frac{\partial}{\partial s}(Q \sin \theta)+p(x)-m \ddot{u}=0, \\
\frac{\partial}{\partial s}(N \sin \theta)+\frac{\partial}{\partial s}(Q \cos \theta)+p(y)-m \ddot{w}=0, \\
\frac{\partial M}{\partial s}-Q=J \frac{\partial \ddot{w}}{\partial s} .
\end{array}\right\} .
$$

In equations (3) (4), $d s^{*}$ is the original length of the microsegment; $\theta$ is the angle between the deformed center line and positive $X$ direction; $u$ and $w$ are the displacements of the microsegments in the $X$ and $Y$ axes, respectively; $p(x)$ and $p(y)$ are external loads along the $X$ and $Y$ axes; $M, N$, and $Q$ are the bending moment, axial force, and shear force on the section, respectively; $\ddot{(})=\partial^{2}() / \partial t^{2}$.

Although the mechanism has not been understood clearly, present test study and existed experimental study [2] have shown that the contents and pressure of pipe can seriously affect its mechanical behavior. To consider the inertial force caused by the contents' mass, the mass per unit length and the moment of inertia in equation (4) are

$$
\left\{\begin{array}{l}
m=\pi \rho_{\mathrm{p}} \frac{D^{2}+\left(\alpha_{\mathrm{p}}-1\right) d^{2}}{4}, \\
J=\pi \rho_{\mathrm{p}} \frac{D^{4}+\left(\alpha_{\mathrm{p}}-1\right) d^{4}}{32}, \\
\alpha_{\mathrm{p}}=\frac{\rho_{\mathrm{m}}}{\rho_{\mathrm{p}}}
\end{array}\right.
$$

where $D$ and $d$ are the outer and inner diameter of the pipe and $\rho_{\mathrm{p}}$ and $\rho_{\mathrm{m}}$ are the density of the pipe and the contents, respectively.

Theoretically, due to large deformation from the intense dynamic loading, axial force exists along with bending moment during the whole period of dynamic response. However, it has been shown [34] that although the cantilever structure undergoes large deformation under dynamic impact, its axial force remains relatively low level and always does not exceed $10 \%$ of the axial yield force. Since the global response of the structure is mainly controlled by bending, the two variables seemed to be uncoupled and independent of each other during the analysis. So, axial force $N$ can be calculated by

$$
N=\int_{A} \sigma d A
$$

Considering the influence of internal pressure, the axial stress $\sigma$ of the pipe section is composed of the stress $\sigma_{\mathrm{m}}$ caused by the initial contents pressure and the stress $\sigma_{\mathrm{I}}$ caused by the external load is given by

$$
\begin{aligned}
\sigma & =\sigma_{\mathrm{m}}+\sigma_{\mathrm{I}}, \\
\sigma_{\mathrm{m}} & =\frac{p_{i} R v}{H}, \\
\sigma_{\mathrm{I}} & =\sigma(\varepsilon), \\
\varepsilon & =\sqrt{\left(1+u^{\prime}\right)^{2}+\left(w^{\prime}\right)^{2}}-1,
\end{aligned}
$$

where $p_{i}, R$, and $v$ are internal pressure, radius, and the pipe's Poisson's ratio, respectively.

Equations (5) and (7a)-(7c) are general expressions to consider the content and pressure. When $d=0$ and $p_{i}=0$, the governing equations degrade to the case of adapting only to solid-section beam. On the other hand, when $\alpha_{\mathrm{p}}=0$ and $p_{i}=0$, the governing equations are only valid for empty pipes, which had been investigated extensively (e.g., Reid et al. [4] and Shaw et al. [35]). 
As discussed in Section 2, in contrast to the bending behavior of solid-section beams, the bending process of thinwalled pipes includes the flattening of the section and softening caused by more complicated partial section collapse $[3,4]$, which frequently involves complex geometry and material nonlinear evolution and is a challenging task to accurately analyze by stress analysis methods [4]. Therefore, the true section $M-\kappa$ curves measured from Section 2 were introduced into the dynamic equation to capture its complex mechanical behavior. Figure 14 shows a simplified $M-\kappa$ curve of a typical pipe section under pure bending. To simplify the analysis, the loading and unloading paths in the numerical calculation were stipulated to comply with the rules described in the following equations:

$$
\begin{aligned}
& M= \begin{cases}f_{\mathrm{e}}(\kappa), & 0<\kappa<\kappa_{\mathrm{e}}, \\
f_{\mathrm{h}}(\kappa), & \kappa_{e}<\kappa<\kappa_{\mathrm{cr}}, \Delta \kappa>0, \\
f_{\mathrm{s}}(\kappa), & \kappa>\kappa_{\mathrm{cr}}, \Delta \kappa \geq 0,\end{cases} \\
& M= \begin{cases}M_{\mathrm{a}}+E I\left(\kappa-\kappa_{\mathrm{a}}\right), \quad \kappa_{\mathrm{a}}-\frac{2 M_{\mathrm{a}}}{\mathrm{EI}}<\kappa<\kappa_{\mathrm{a}}, \\
-f_{\mathrm{h}}\left(\kappa_{\mathrm{a}}+\left|\kappa-\kappa_{\mathrm{b}}\right|\right), \\
-f_{\mathrm{s}}\left(\kappa_{\mathrm{a}}+\left|\kappa-\kappa_{\mathrm{b}}\right|\right), & \kappa<\kappa_{\mathrm{a}}-\frac{2 M_{\mathrm{a}}}{\mathrm{EI}}, \Delta \kappa<0,\end{cases} \\
& \kappa_{\mathrm{b}}=\kappa_{\mathrm{a}}-\frac{2 M_{\mathrm{a}}}{\mathrm{EI}},
\end{aligned}
$$

where $\kappa_{\mathrm{e}}$ and $\kappa_{\mathrm{cr}}$ are critical curvatures corresponding to the three stages of elasticity, plastic hardening, and softening; $\kappa_{\mathrm{a}}$ and $M_{\mathrm{a}}$ are the sectional curvature and bending moment at the start of unloading. $f_{\mathrm{h}}(\kappa)$ and $f_{\mathrm{s}}(\kappa)$ are nonlinear relationships between moment and curvature measured from the quasistatic test.

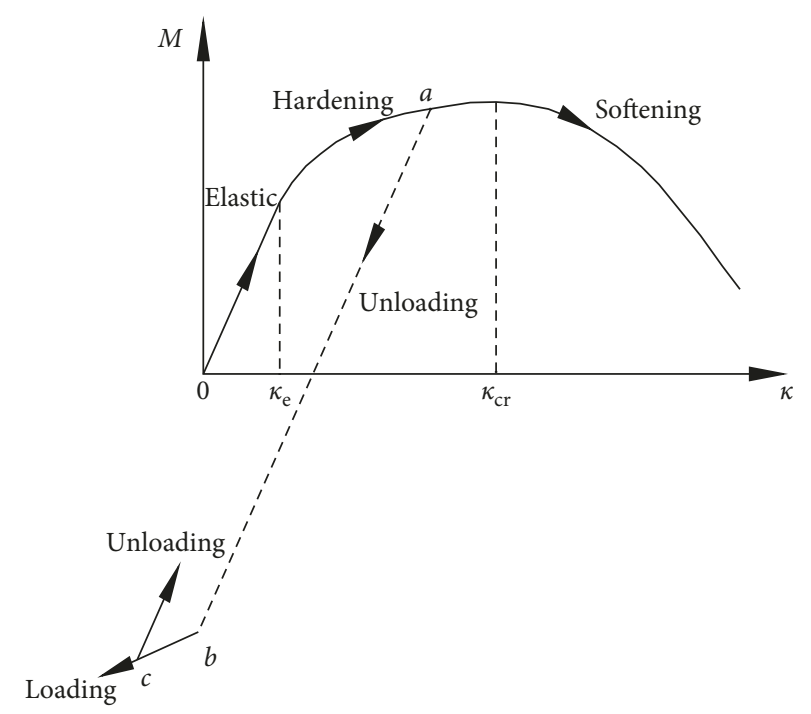

FIgURE 14: $M-\kappa$ curve for elastic, hardening-softening behavior of the pressurized pipe.

To solve the above equations, the following boundary conditions are introduced:

$$
\begin{cases}Q=F(t), M=0, & x=L, \\ w=0, w^{\prime}=0, & x=0, \\ w(x, t)=0, \dot{w}(x, t)=0, p_{i}=p, & t=0 .\end{cases}
$$

3.2. Discrete Form of Governing Equations and Solutions. To solve equation (4), the cantilever pipe shown in Figure 12 is equally divided into $n$ microsegments in the axial direction, as shown in Figure 15.

The discrete form of the governing equation (4) is

$$
\left.\begin{array}{rl} 
& \left(N_{i+1} \cos \theta_{i+1}-N_{i} \cos \theta_{i}\right)-\left[\frac{M_{i+1}-M_{i}}{d s_{i+1}} \sin \theta_{i+1}-\frac{M_{i}-M_{i-1}}{d s_{i}} \sin \theta_{i}\right] \\
+ & {\left[\frac{\ddot{w}_{i+1}-\ddot{w}_{i}}{d s_{i+1}} \sin \theta_{i+1}-\frac{\ddot{w}_{i}-\ddot{w}_{i-1}}{d s_{i}} \sin \theta_{i}\right]+p(x)\left[\frac{d s_{i}+d s_{i+1}}{2}\right]-m_{i} \ddot{u}_{i}=0,} \\
& \left(N_{i+1} \sin \theta_{i+1}-N_{i} \sin \theta_{i}\right)+\left[\frac{M_{i+1}-M_{i}}{d s_{i+1}} \cos \theta_{i+1}-\frac{M_{i}-M_{i-1}}{d s_{i}} \cos \theta_{i}\right] \\
- & J\left[\frac{\ddot{w}_{i+1}-\ddot{w}_{i}}{d s_{i+1}} \cos \theta_{i+1}-\frac{\ddot{w}_{i}-\ddot{w}_{i-1}}{d s_{i}} \cos \theta_{i}\right]+p(y)\left[\frac{d s_{i}+d s_{i+1}}{2}\right]-m_{i} \ddot{w}_{i}=0,
\end{array}\right\} \quad(i=0,1, \ldots, n-1) .
$$




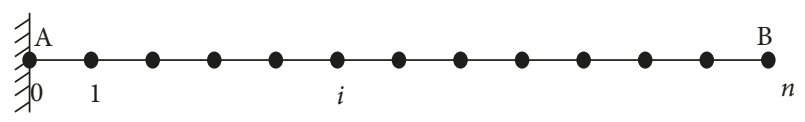

FIgURE 15: Discrete model of cantilever pressurized pipe.

In which

$$
\left\{\begin{array}{l}
d s_{i}=\sqrt{\left(1+\frac{u_{i+1}-u_{i}}{d s_{i}^{*}}\right)^{2}+\left(\frac{w_{i+1}-w_{i}}{d s_{i}^{*}}\right)^{2}} \cdot d s_{i}^{*}, \\
\sin \theta_{i}=\frac{w_{i+1}-w_{i}}{\sqrt{\left(d s_{i}^{*}+u_{i+1}-u_{i}\right)^{2}+\left(w_{i+1}-w_{i}\right)^{2}}}, \quad(i=1, \ldots, n), \\
\cos \theta_{i}=\frac{d s_{i}^{*}+u_{i+1}-u_{i}}{\sqrt{\left(d s_{i}^{*}+u_{i+1}-u_{i}\right)^{2}+\left(w_{i+1}-w_{i}\right)^{2}}},
\end{array}\right.
$$

where $d s_{i}$ is the length of the $i$ microsegment after deformation.

Equation (10) is a general discrete form of governing equation for both empty pipe, pressurized pipe, and even solid-section beam. By bringing the $M-\kappa$ relationships measured from quasistatic test (e.g., Figures 9-11) to equation (10), the transient response of pipe filled with contents and pressure can be predicated numerically. It should be noted that the change of pressure during the dynamic process may be an important issue. It has been reflected on the used experimental $M-\kappa$ curve during the quasistatic test; the recorded evolution of internal pressure is expected to occur during the dynamic response (see Figure 8$)$. In addition, as the impact velocity is low $(<10 \mathrm{~m} / \mathrm{s})$, the plastic hinge and local collapse of the pipe should not be too different between quasistatic and dynamic loading cases which legitimate the above assumption. Also, flow speed in the pipe is much smaller than the acoustic wave speed, which supports the assumption of using a quasistatic method to deal with $M-\kappa$ relationship. Consequently, the conclusions obtained in this study are expected to be valid to a wide range of low-velocity impact behavior of the pressurized pipe. However, this does not prevent the use of dynamic experimentally determined $M-\kappa$ relationship for further simulation if they are available.

The discrete form of axial strain and section curvature is

$$
\begin{aligned}
& \varepsilon_{i}=\sqrt{\left(1+\frac{u_{i+1}-u_{i}}{d s_{i}^{*}}\right)^{2}+\left(\frac{w_{i+1}-w_{i}}{d s_{i}^{*}}\right)^{2}}-1, \\
& \kappa_{i}=\frac{\left(d s_{i}^{*}+u_{i+1}-u_{i}\right)\left(w_{i+1}-2 w_{i}+w_{i-1}\right)-\left(w_{i+1}-w_{i}\right)\left(u_{i+1}-2 u_{i}+u_{i-1}\right)}{\left[\left(w_{i+1}-w_{i}\right)^{2}+\left(d s_{i}^{*}+u_{i+1}-u_{i}\right)^{2}\right]^{3 / 2}} .
\end{aligned}
$$

Considering the boundary conditions in equation (9), the equation can be solved by discretizing time. Assuming that at the instant $t^{q}$, the accelerations $\ddot{u}_{i}^{q}$ and $\ddot{w}_{i}^{q}$ of the node $i$ are known, the displacements $u_{i}^{q+1}$ and $w_{i}^{q+1}$ at the next instant $t^{q+1}$ can be obtained by applying

$$
\left.\begin{array}{l}
u_{i}^{q+1}=\ddot{u}_{i}^{q}(\Delta t)^{2}+2 u_{i}^{q}-u_{i}^{q-1}, \\
w_{i}^{q+1}=\ddot{w}_{i}^{q}(\Delta t)^{2}+2 w_{i}^{q}-w_{i}^{q-1} .
\end{array}\right\} .
$$

Applying the obtained displacements $u_{i}^{q+1}$ and $w_{i}^{q+1}$, one can calculate strain $\varepsilon_{i}$ and curvature $\kappa_{i}$ at instant $t^{q+1}$ based on equations (12) and (13). Axial force and moment are then calculated according to the corresponding constitutive relation (e.g., equations (6), (7a), (7b), (7c), (8a), (8b), and (8c)). In addition, after obtaining moment and axial force, $\ddot{u}_{i}^{q+1}$ and $\ddot{w}_{i}^{q+1}$ are obtained from the equation of motion (e.g., equation (14)). In this way, the elastoplastic dynamic response history of a pressurized cantilever pipe can be predicted.

The whipping of empty pipe is always linked with the formation of one or several plastic hinges (kinks) along its span [4]. In such cases, the numerical solutions through central difference may exhibit considerable fluctuations in space and time. The moving-least-square-reproducingkernel-particle method (MLSRKPM) developed by Shaw et al. [35] has a superior performance to arrest these numerical fluctuations through a suitable choice of window size. Readers could refer to [35] for more information.

\section{Numerical Results and Discussions}

The rationality and validity of the test data measured in Section 2 and the numerical method described in Section 3 will be demonstrated in this section.

4.1. Loading and Simulation Parameters. To numerically investigate the deformation mechanism of an elastoplastic cantilever pressurized pipe, the specimen corresponding to the previous pure bending experiments is selected to analyze in present simulations.

Based on the experimental observation described in Section 2, the empty pipe, a sand-filled pipe (without internal pressure), and a water-filled (internal pressure $15 \mathrm{MPa}$ ) pipe are selected for numerical analysis. The critical curvatures for plastic hardening and softening behavior are $1.25 \mathrm{~m}^{-1}, 3.2 \mathrm{~m}^{-1}$, and $4.0 \mathrm{~m}^{-1}$, respectively. Since there are limited experimental publications on cantilever pipes under 
impact loads, the present simulation uses the endconcentration force measured by the pipe-whip experiment conducted by Reid et al. [4] as an impact load. The time history is shown in Figure 16. In addition, to account for the mass of the impact block, a mass of $1.0 \mathrm{~kg}$ is concentrated on the cantilever tip in the numerical calculation. For stability [34], $n=41$ and $\Delta t=5.0 \mu$ s are used for all present simulations after a sensitivity check.

4.2. Dynamic Mechanism for Empty Pipe. Figure 17 shows the deformed configurations of the empty pipe at different instants when subjected to a concentrated impact at its tip. Generally, it shows similar global deformation modes as in solid-section cantilever beam observed by Reid and Gui [36], i.e., the transient flexural vibration prorogates along the beam and followed by a large plastic rotation around its fixed end. However, marked difference can be easily detected immediately after the impact occurs. At instant $5 \mathrm{~ms}$, the first kink is formed at $k_{1}$ section, which is about $0.275 \mathrm{~m}$ from the impact tip, and hints for local collapse occur at this position. Then, the second and third kinks are sequentially formed at about $10 \mathrm{~ms}$ and $15 \mathrm{~ms}$, which are located in $0.50 \mathrm{~m}$ and $0.875 \mathrm{~m}$ from its tip. Visually, almost during the entire response process, the whole pipe is divided into four separated segment by these kinks.

The curvature distribution shown in Figure 18 supports the above findings. At $5.0 \mathrm{~ms}$, the curvature at $k_{1}$ exceeds the critical curvature, i.e., $1.25 \mathrm{~m}^{-1}$ of the pipe, indicating that the section has collapsed due to bending and formed a kink here. As the response progresses, kinks also form at $k_{2}$ and $k_{3}$ positions, at $0.50 \mathrm{~m}$ and $0.875 \mathrm{~m}$ from the tip, which is exactly the same as described from the deformation configuration. The segments adjacent to the kink rotate around it and consume impact energy.

The local collapse of the cross section makes the whole response process cover three distinct stages of initial elasticity, medium-term plastic hardening, and postsoftening, which is evidently different from the mechanism for solidsection cantilever beam discussed by Reid and Gui [36].

In addition, the dissipation of energy through internal plastic work in several typical sections is illustrated in Figure 19, which provides a basis for comparison with the results of the elastic-plastic analysis of beam with a solid section. It was revealed that the sections at $k 1, k 2$, and $k 3$ and root dominate the dissipation energy of pipe sequentially along the whole phase of the transient response. Generally, these sections dissipate approximately $95 \%$ of the whole plastic work, in which, however, almost $64.12 \%$ of the available kinetic energy is dissipated in the interior kink positions compared to $30.25 \%$ as absorbed at the root of the pipe. These differ markedly from the findings reported by Reid and Gui [36] about the solid section's beam in which root rotation dominates the overall behavior of the beams and consumes the vast majority of external energy.

4.3. Influence of Contents and Internal Pressure. To explore the effects of internal pressure and content, Figure 20 demonstrates the deformation curves of the pipe with

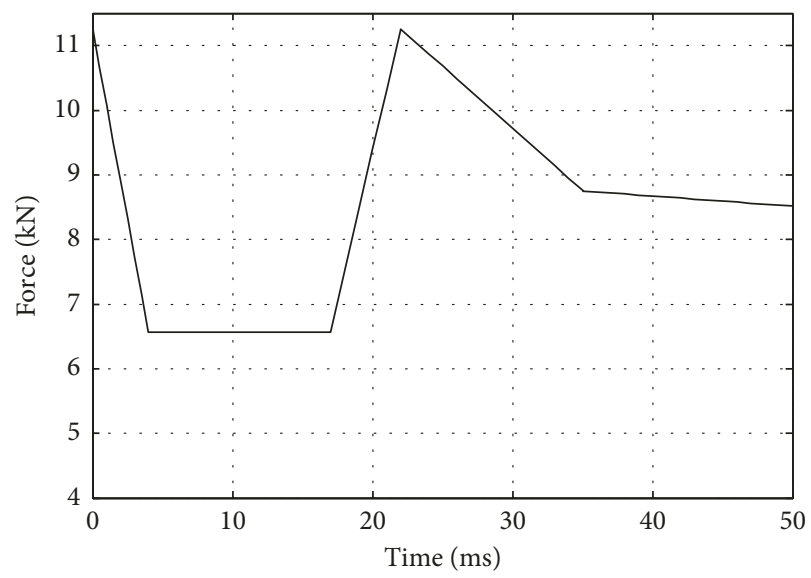

FIGURE 16: Time history of impact force loaded at the tip of pipe [4].

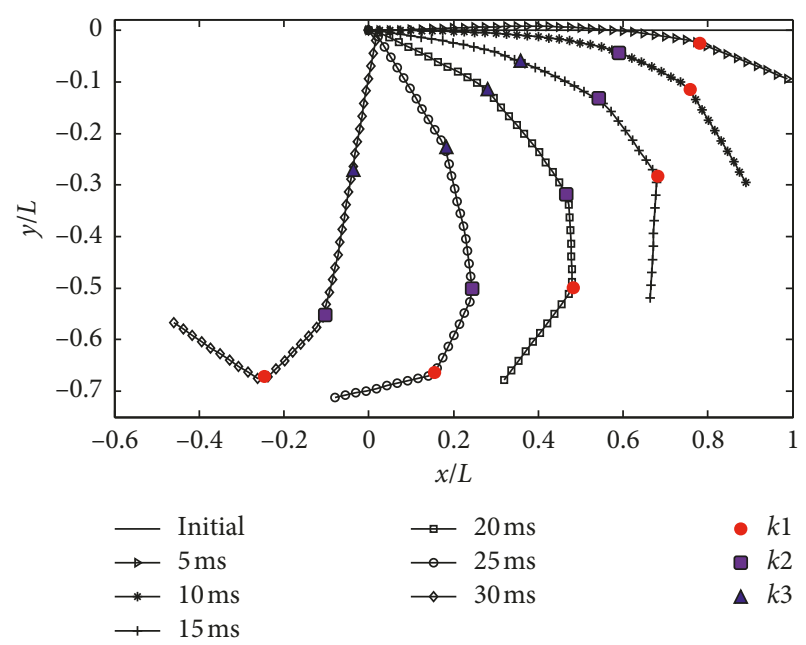

FIgURE 17: Deformed configurations at various instants for the empty pipe.

water (internal pressure $15 \mathrm{MPa}$ ) and sand under impact load. Similar to the analysis of the solid-section rectangular beam in the literature [34] but contrary to the findings in Section 4.2, the integral pipe section remains smooth and continuous during the whole plastic dynamic response process. It exhibits the distinctive characteristics of the transition hinge stage of early bending, plastic hinge from the end to the root movement, and the dominant structure response of late root stationary hinge. Compared to Figure 17, there are not any kinks formed as exhibited by empty pipes in either case.

Upon examining the curvature distribution curves shown in Figure 21, it was found that except for at the fixed end, the pipes do not exceed their critical curvatures $3.2 \mathrm{~m}^{-1}$ and $4.0 \mathrm{~m}^{-1}$, respectively, although some sections are approaching. This shows that except for the fixed end section, the other sections only experience early elasticity and medium-term plastic hardening processes, while the softening stage of the empty pipe does not appear.

It is worth noting that the impact load exerted on the cantilever pipe is same for these three pipes; the absence of 


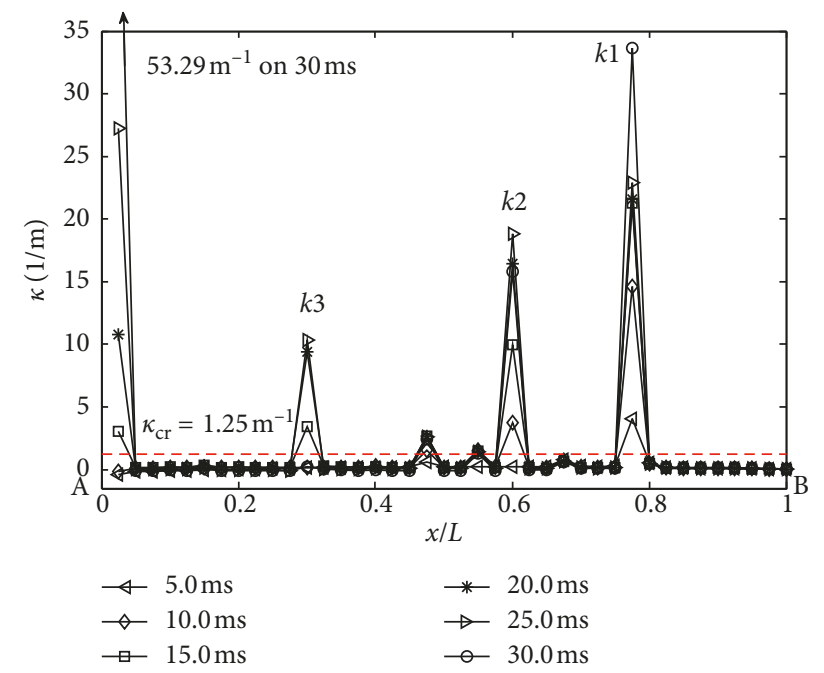

FIGURE 18: Curvature distributions at various instants for the empty pipe.

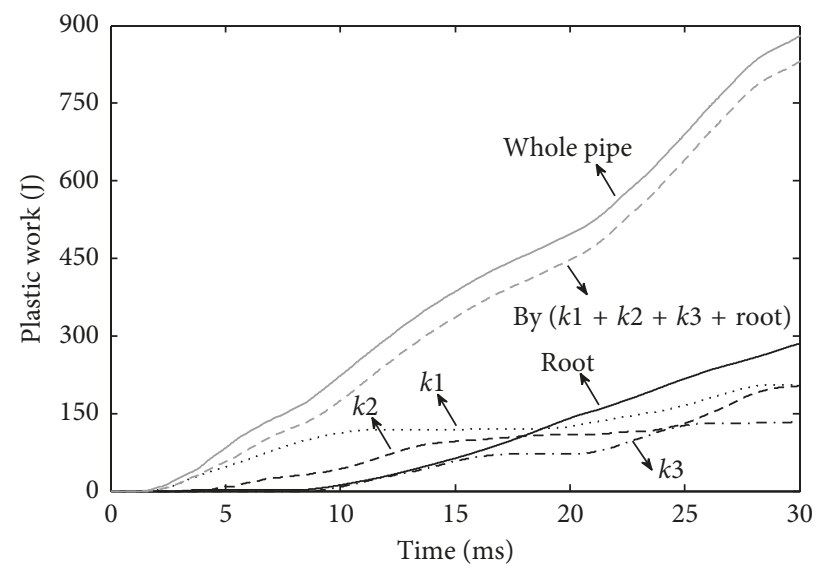

Figure 19: Time histories of energy dissipation for the whole empty pipe and root and "kink" sections shown in Figure 18.

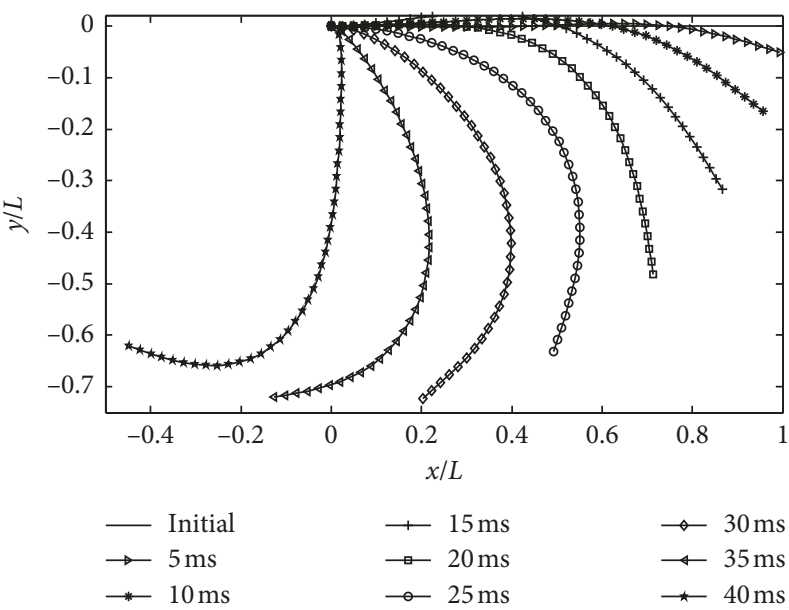

(a)

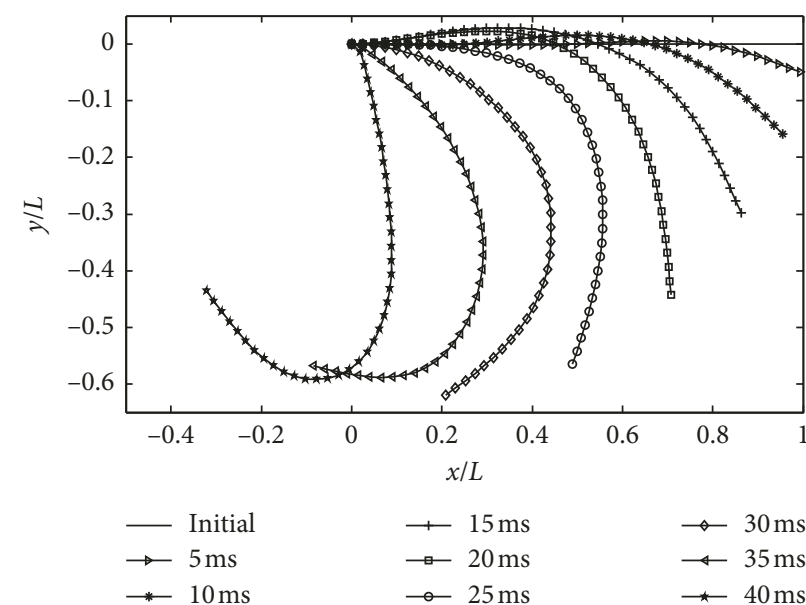

(b)

FIgURe 20: Deformed configurations at various stages: (a) filled by water with pressure of $15 \mathrm{MPa}$ and (b) filled with sand. 


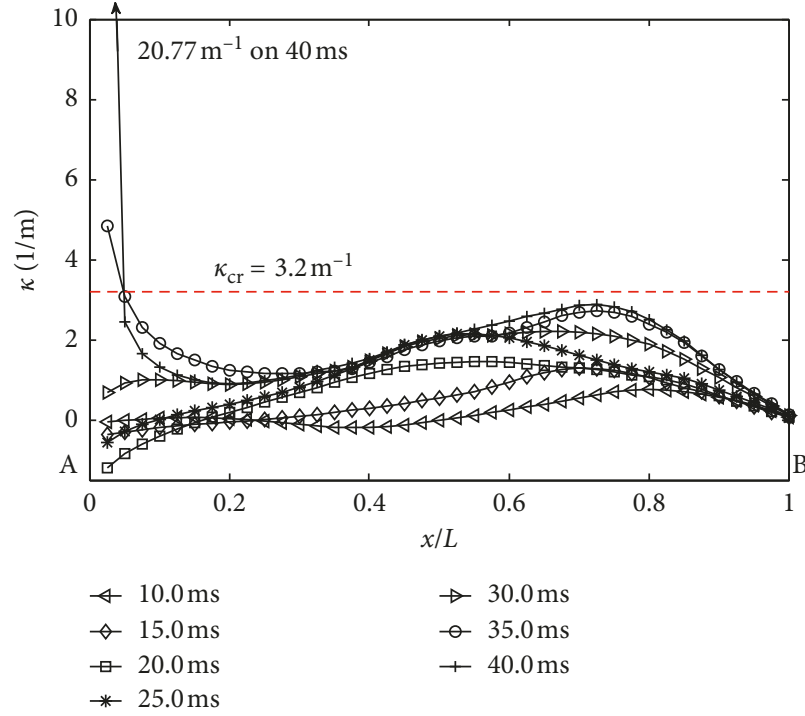

(a)

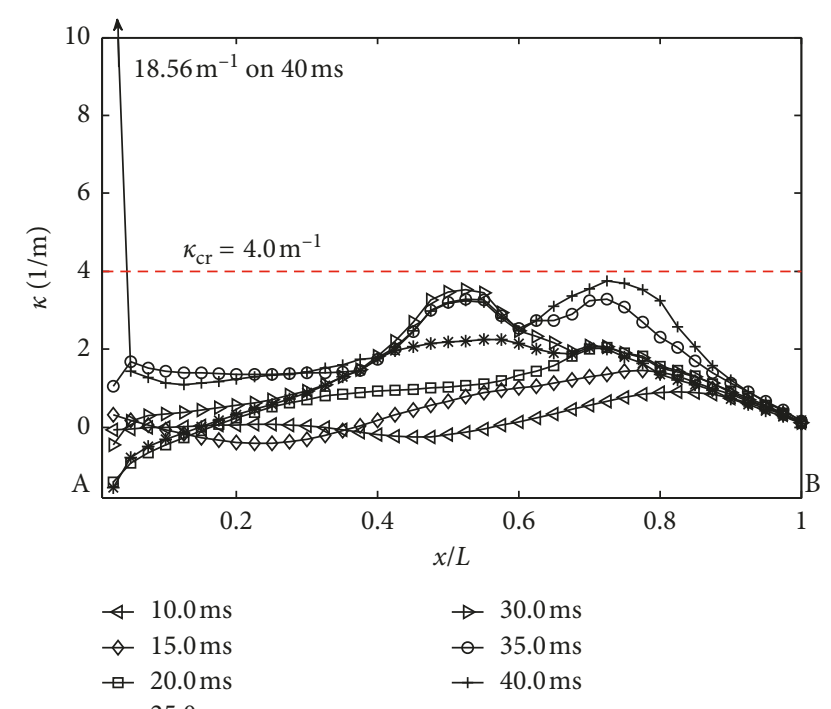

(b)

FIgURE 21: Curvature distributions at various instants: (a) filled by water with pressure of $15 \mathrm{MPa}$ and (b) filled with sand.

the kink in the latter two pipes implies that their high flexural capacity impedes the occurrence the local failure in their interior section and make their flexural behavior more approaching the one of the solid section beams.

Figure 22 is analogous to Figure 19 in showing the evolution of plastic work in the pipes. However, there are some notable differences. First, the distribution of plastic work implies that the majority of the energy is not absorbed separately along the pipe but concentrated at the root. For pipe with sand and water $(15 \mathrm{MPa})$, of the $1188 \mathrm{~J}$ and $1012 \mathrm{~J}$ dissipated, about $614.7 \mathrm{~J}(51.74 \%)$ and $556.4 \mathrm{~J}(54.98 \%)$ are absorbed in the element at the root compared with $30 \%$ in the empty pipe. Furthermore, the distribution approaches more closely with one which is predicted by the elasticplastic-analysis on the beam with a solid section. Meanwhile, the difference in energy dissipation ratio, e.g., $51.74 \%$ and $54.98 \%$ for present analysis and $87 \%$ given by Reid and Gui [36] for solid-section beam, verifies the significant role of the local deformation about the pipe.

The difference in the numerical responses of the pressurized and empty pipes reflects the influence of the contents and pressure, which promotes the critical curvature and thus improves the global plastic flexural capacity.

\section{Conclusions}

This study carried out pure bending experiments on pressurized pipes. By comparing the deformed configuration and $M-\kappa$ curves of the pipes, the influence of pipe contents and its initial internal pressure on the pure bending mechanical behavior of the thin-walled circular tube was identified. It was found that the thin-walled empty pipe exhibits obvious elastic-plastic hardening-softening behavior under bending. When the curvature exceeds the critical curvature $\kappa_{\mathrm{cr}}$, a distinct deformation kink forms on shorter partial pipe sections in the interior of the pure curved section. The

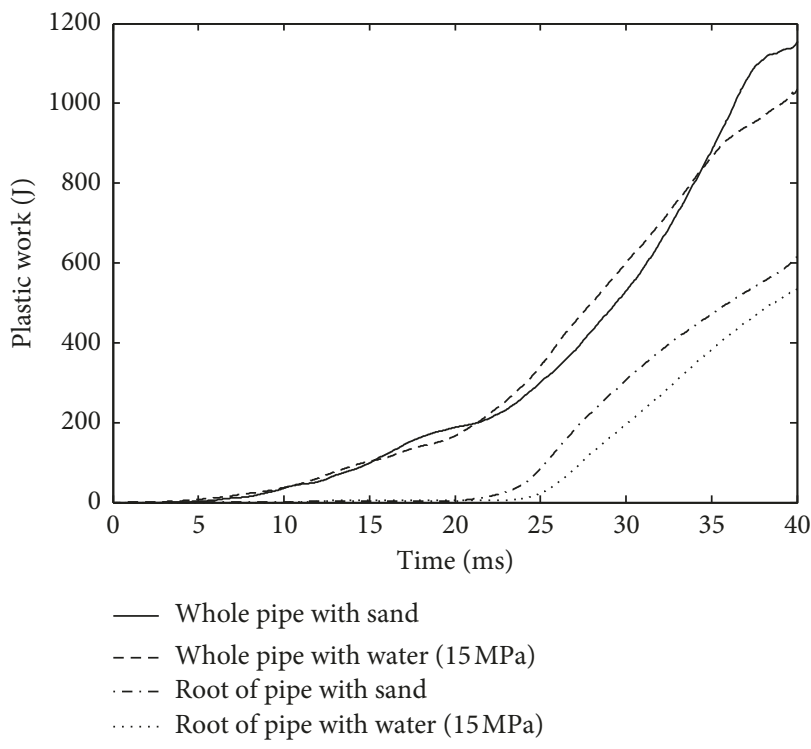

FIGURE 22: Time histories of energy dissipation for whole pipe and root section.

critical curvature and peak moment will increase significantly with increasing internal pressure. Even if the pipe is filled with no pressure, the critical curvature and peak bending moment will be significantly improved compared with an empty pipe. When the contents are water and air, the bending behavior of the pipe is different even under the same internal pressure, i.e., the hydrodynamic properties of the pipe contents are important factors affecting the bending behavior of the pipe. Together with internal pressure, it determines the amplitude of the pipe's bending resistance. At the same time, it was also found that internal sand will effectively improve the ability of the pipe to maintain a large bending deformation and shift its behavior approaching one with solid section. 
Then, $M-\kappa$ curves were used to describe the bending behavior of the pipe-whip. Based on the postdeformation configuration, the governing equations were derived and the numerical simulations were conducted to understand the transient dynamic response to low-velocity impact of the cantilever pressurized pipe. The numerical result showed that the present method can predict the dynamic features of a pressurized pipe with high precision. By comparing the shape of pipe after deformation and the distribution of curvature along the length of pipe, the effects of contents and pressure on its transient dynamic behavior of the component were investigated. Comparatively, the thin-walled empty pipe exhibits obvious elastic-plastic hardening-softening behavior, and some sections will fail as they form kinks during the whole response. However, the pipe contents and internal pressure can effectively resist local flattening and failure of the section, increase the critical curvature of the section softening, and then impede the occurrence of the local collapse of the pipe.

It is necessary to point out that the cantilever pipe studied in this paper has a low axial force during the dynamic response process even if large deformation occurs due to the bearing boundary conditions. Therefore, it is feasible to uncouple the bending moment and axial force to simplify the calculation. For a pipe fixed at both ends, large axial forces generated during the response will significantly affect the distribution of bending moments in the pipe section and may dominate the response of the structure in its later stages, rendering the corresponding control equations no longer applicable. Future research should investigate the interaction between bending moment and axial force and derive a simple beam element model that can accurately describe both the local and global response behavior.

\section{Data Availability}

The data used to support the findings of this study are included within the article.

\section{Conflicts of Interest}

The authors declare that there are no conflicts of interest regarding the publication of this paper.

\section{Acknowledgments}

Support from the Natural Science Foundation of China (grant nos. 11672165 and 51208289) is acknowledged.

\section{References}

[1] S. Y. Zhang, G. Y. Lu, G. Q. Cheng et al., "The advances of research on the impact damage and failure of empty and halffilled tubes," Advances in Mechanics, vol. 34, no. 1, pp. 23-31, 2004, in Chinese.

[2] N. Jones and R. S. Birch, "Low-velocity impact of pressurised pipelines," International Journal of Impact Engineering, vol. 37, no. 2, pp. 207-219, 2010.

[3] T. X. Yu, S. R. Reid, and B. Wang, "Hardening-softening behaviour of tubular cantilever beams," International Journal of Mechanical Sciences, vol. 35, no. 12, pp. 1021-1033, 1993.
[4] S. R. Reid, T. X. Yu, J. L. Yang, and G. G. Corbett, "Dynamic elastic-plastic behavior of whipping pipes: experiments and theoretical model," International Journal of Impact Engineering, vol. 18, no. 7-8, pp. 703-733, 1996.

[5] S. R. Reid, B. Wang, and M. Aleyassin, "Structural modeling and testing of failed high energy pipe runs: 2D and 3D pipe whip," International Journal of Pressure Vessels and Piping, vol. 88, no. 5-7, pp. 189-197, 2011.

[6] N. K. Prinja and N. R. Chitkara, "Post-collapse cross-sectional flattening of thick pipes in plastic bending," Nuclear Engineering and Design, vol. 83, no. 1, pp. 113-121, 1984.

[7] B. D. Reddy, "Plastic buckling of a cylindrical shell in pure bending," International Journal of Mechanical Sciences, vol. 21, no. 11, pp. 671-679, 1979.

[8] B. D. Reddy, "An experimental study of the plastic buckling of circular cylinders in pure bending," International Journal of Solids and Structures, vol. 15, no. 9, pp. 669-683, 1979.

[9] S. Gellin, "The plastic buckling of long cylindrical shells under pure bending," International Journal of Solids and Structures, vol. 16, no. 5, pp. 397-407, 1980.

[10] D. Munz and C. Mattheck, "Cross-sectional flattening of pipes subjected to bending," International Journal of Pressure Vessels and Piping, vol. 10, no. 6, pp. 421-429, 1982.

[11] N. K. Prinja and N. R. Chitkara, "Finite-element analyses of post-collapse plastic bending of thick pipes," Nuclear Engineering and Design, vol. 91, no. 1, pp. 1-12, 1986.

[12] L. C. Zhang and T. X. Yu, "An investigation of the brazier effect of a cylindrical tube under pure elastic-plastic bending," International Journal of Pressure Vessels and Piping, vol. 30, no. 2, pp. 77-86, 1987.

[13] S. Kyriakides and G. T. Ju, "Bifurcation and localization instabilities in cylindrical shells under bending-I. Experiments," International Journal of Solids and Structures, vol. 29, no. 9, pp. 1117-1142, 1992.

[14] G. T. Ju and S. Kyriakides, "Bifurcation and localization instabilities in cylindrical shells under bending-II. Predictions," International Journal of Solids and Structures, vol. 29, no. 9, pp. 1143-1171, 1992.

[15] A. Limam, L. H. Lee, E. Corona et al., "Plastic buckling and collapse of tubes under bending and internal pressure," in Proceedings of the Offshore Mechanics and Arctic Engineering Portugal, OMAE08-57986, pp. 675-683, 2015.

[16] A. Limam, L.-H. Lee, E. Corona, and S. Kyriakides, "Inelastic wrinkling and collapse of tubes under combined bending and internal pressure," International Journal of Mechanical Sciences, vol. 52, no. 5, pp. 637-647, 2010.

[17] S. Houliara and S. A. Karamanos, "Buckling and postbuckling of long pressurized elastic thin-walled tubes under in-plane bending," International Journal of Non-Linear Mechanics, vol. 41, no. 4, pp. 491-511, 2006.

[18] C. Mathon and A. Limam, "Experimental collapse of thin cylindrical shells submitted to internal pressure and pure bending," Thin-Walled Structures, vol. 44, no. 1, pp. 39-50, 2006.

[19] S. R. Reid and K. Goudie, "Denting and bending of tubular beams under local loads," in Structural Failure, T. Wierzbicki and N. Jones, Eds., pp. 331-364, Wiley-Interscience, John Wiley and Sons, New York, NY, USA, 1989.

[20] N. Jones and W. Q. Shen, "A theoretical study of the lateral impact of fully clamped pipelines," Proceedings of the Institution of Mechanical Engineers, vol. 206, no. 2, pp. 129-146, 1992.

[21] A. Palmer, A. Neilson, and S. Sivadasan, "Impact resistance of pipelines and the loss-of-containment limit state," Journal of Pipeline Integrity, vol. 2, no. 4, pp. 231-240, 2003. 
[22] W. Johnson, S. K. Ghosh, A. G. Mamalis, T. Y. Reddy, and S. R. Reid, "The quasi-static piercing of cylindrical tubes or shells," International Journal of Mechanical Sciences, vol. 22, no. 1, pp. 9-20, 1980.

[23] G. G. Corbett, S. R. Reid, and S. T. S. Al-Hassani, "Static and dynamic penetration of steel tubes by hemispherically nosed punches," International Journal of Impact Engineering, vol. 9, no. 2, pp. 165-190, 1990.

[24] N. Jones and R. S. Birch, "Influence of internal pressure on the impact behavior of steel pipelines," Journal of Pressure Vessel Technology, vol. 118, no. 4, pp. 464-471, 1996.

[25] L. E. Schwer, B. S. Holmes, and S. W. Kirkpatrick, "Response and failure of metal tanks from impulsive spot loading: experiments and calculations," International Journal of Solids and Structures, vol. 24, no. 8, pp. 817-833, 1988.

[26] N. Jones, S. E. Birch, R. S. Birch, L. Zhu, and M. Brown, "An experimental study on the lateral impact of fully clamped mild steel pipes," Proceedings of the Institution of Mechanical Engineers, vol. 206, no. 2, pp. 111-127, 1992.

[27] A. Palmer, A. Neilson, and S. Sivadasan, "Pipe perforation by medium-velocity impact," International Journal of Impact Engineering, vol. 32, no. 7, pp. 1145-1157, 2006.

[28] M. Nishida and K. Tanaka, "Experimental study of perforation and cracking of water-filled aluminum tubes impacted by steel spheres," International Journal of Impact Engineering, vol. 32, no. 12, pp. 2000-2016, 2006.

[29] K. Chen and W. Q. Shen, "Further experimental study on the failure of fully clamped steel pipes," International Journal of Impact Engineering, vol. 21, no. 3, pp. 177-202, 1998.

[30] Q. H. Shah, "Experimental and numerical study on the orthogonal and oblique impact on water filled pipes," International Journal of Impact Engineering, vol. 38, no. 5, pp. 330-338, 2011.

[31] G. Y. Lu, S. Y. Zhang, J. P. Lei, and J. L. Yang, "Dynamic responses and damages of water-filled pre-pressurized metal tube impacted by mass," International Journal of Impact Engineering, vol. 34, no. 10, pp. 1594-1601, 2007.

[32] G. Y. Lu, J. P. Lei, Y. Z. Wu et al., "Experimental research on failure of multi-span thin-walled pressure pipelines impacted by projectiles," Explosion and Shock Waves, vol. 23, no. 5, pp. 454-459, 2003, in Chinese.

[33] S. R. Reid, T. X. Yu, and J. L. Yang, "Hardening-softening behaviour of circular pipes under bending and tension," International Journal of Mechanical Sciences, vol. 36, no. 12, pp. 1073-1085, 1994.

[34] F. Xi, F. Liu, and Q. M. Li, "Large deflection response of an elastic, perfectly plastic cantilever beam subjected to a step loading," International Journal of Impact Engineering, vol. 48, pp. 33-45, 2012.

[35] A. Shaw, D. Roy, S. R. Reid, and M. Aleyaasin, "Reproducing kernel collocation method applied to the non-linear dynamics of pipe whip in a plane," International Journal of Impact Engineering, vol. 34, no. 10, pp. 1637-1654, 2007.

[36] S. R. Reid and X. G. Gui, "On the elastic-plastic deformation of cantilever beams subjected to tip impact," International Journal of Impact Engineering, vol. 6, no. 2, pp. 109-127, 1987. 


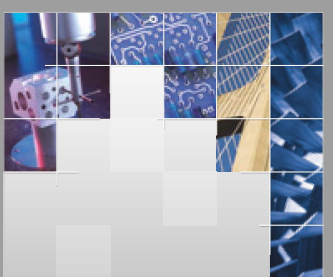

\section{Enfincering}
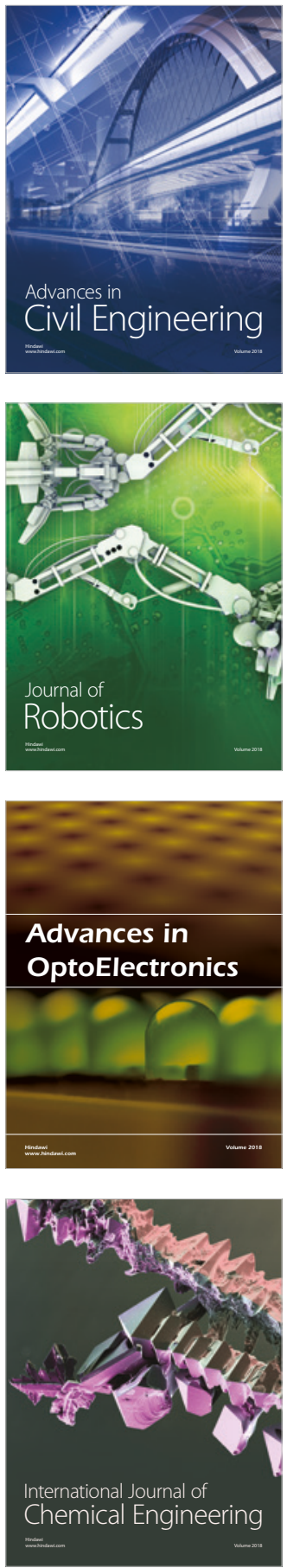

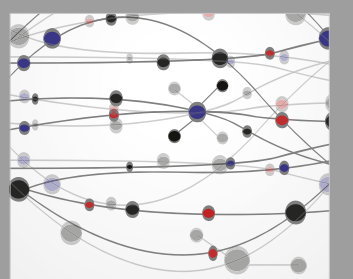

\section{Rotating \\ Machinery}

The Scientific World Journal

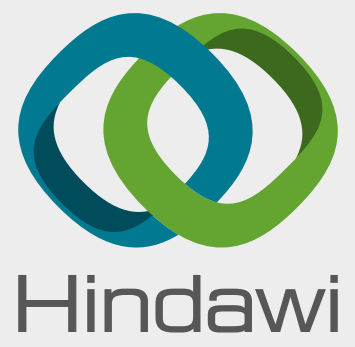

Submit your manuscripts at

www.hindawi.com
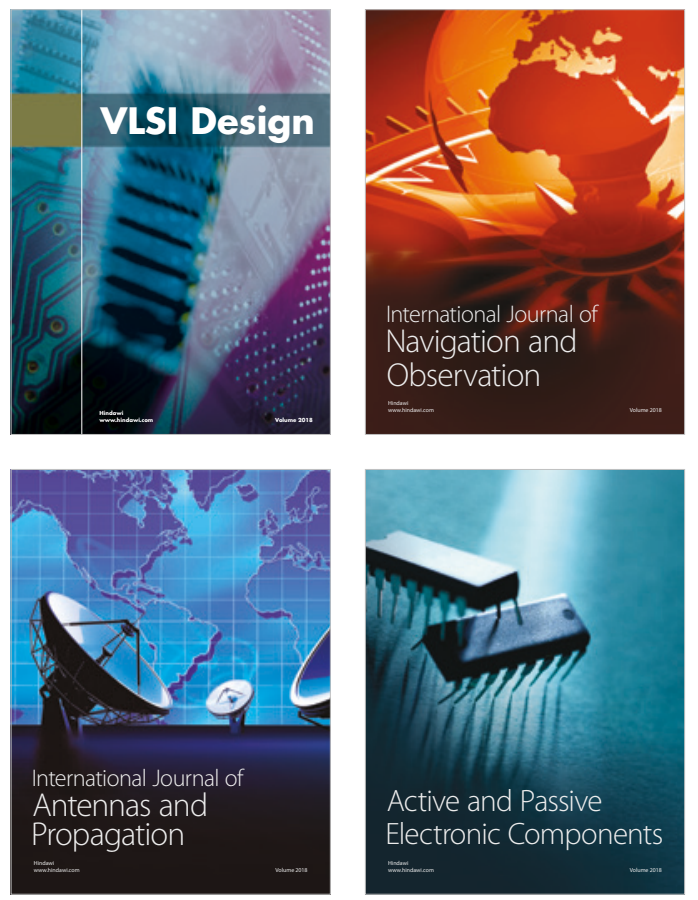
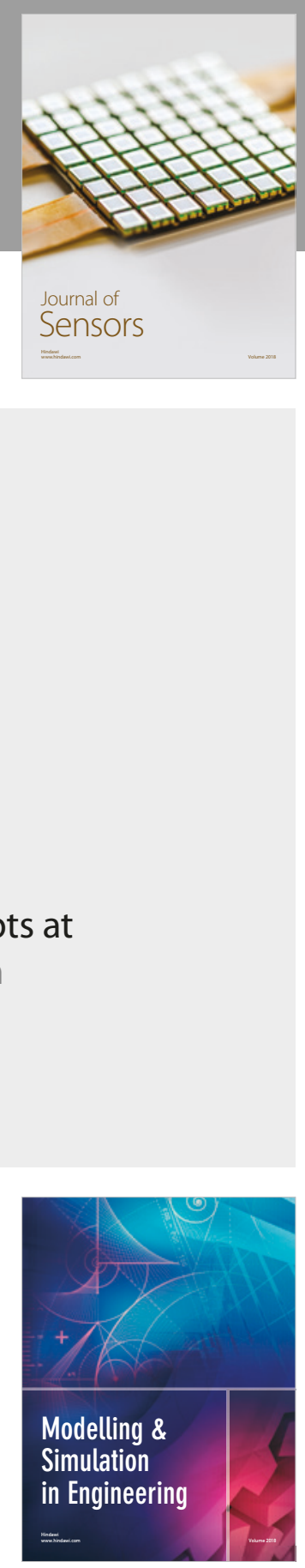

\section{Advances \\ Multimedia}
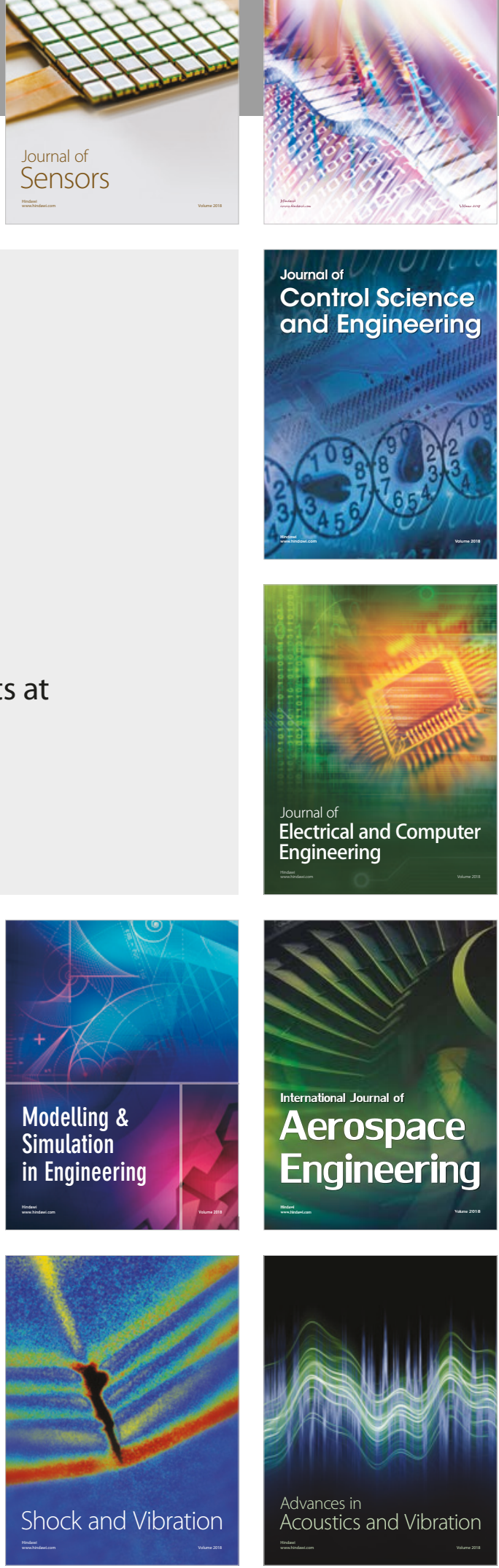